\title{
ESTUDO DAS CONDIÇÕES DE SAÚDE DAS CRIANÇAS NO MUNICÍPIO DE SÃO PAULO, SP (BRASIL), 1984-1985
}

\author{
V - ANEMIA*
}

Carlos Augusto Monteiro**

Sophia Cornbluth Szarfarc**

MONTEIRO, C.A. \& SZARFARC, S. C. Estudo das condições de saúde das crianças no Município de São Paulo, SP (Brasil), 1984-1985. V - Anemia. Rev. Saúde públ., S. Paulo, 21:255-60, 1987.

RESUMO: Dentro de estudo populacional sobre condições de saúde na infância, uma amostra representativa das crianças menores de cinco anos do Município de São Paulo, Brasil $(n=912)$ foi estudada quanto à prevalência e distribuição da anemia. A freqüência de crianças anêmicas (concentração de hemoglobina < $11 \mathrm{~g} / \mathrm{d} 1$ ) foi de $35,6 \%$, sendo de $14,7 \%$ a freqüência de casos severos (hemoglobina $<9,5 \mathrm{~g} / \mathrm{d} 1$ ). As maiores prevalências de anemia se concentraram na faixa etária de 6 a 24 meses de idade, onde mais da metade das crianças se mostraram anêmicas e cerca de metade dos casos corresponderam a formas severas da deficiência. Embora a prevalência da anemia tenda a diminuir com a melhoria do nível socioeconômico da população, não se detectaram estratos da população imunes à deficiência. Em comparação à situação observada no Município há cerca de dez anos, verifica-se elevação expressiva da prevalência da anemia: mais de $50 \%$ para o total de casos e mais de $100 \%$ para as formas severas da deficiência. O conjunto das informações geradas pelo estudo epidemiológico realizado em 1984/85 sugere que a anemia na infância no Município de São Paulo esteja relacionada basicamente a características desfavoráveis da alimentação infantil, sendo menos relevante o papel que poderia ser atribuído às parasitoses intestinais.

UNITERMOS: Saúde da criança. Inquéritos epidemiológicos. Anemia, incidência. Anemia ferropriva, incidência

A anemia - ou o estado decorrente da incapacidade do tecido eritropoiético manter normal a concentração de hemoglobina - é problema de ocorrência endêmica em países do Terceiro Mundo. Determinado na grande maioria das vezes pela deficiência de ferro, o estado anêmico representa considerável agravo à saúde, estando a ele associados prejuízos não só à capacidade produtiva dos indivíduos, como também a importantes aspectos da reprodução, do desenvolvimento cognitivo e da imunocompetência ${ }^{5,15,16}$.

São poucos e dispersos os estudos sobre ocorrência de anemia realizados no Brasil, a maioria deles referindo-se a grupos populacionais restritos estudados à base de amostras de pequenas localidades ou de clientelas de serviços de saúde ${ }^{7,18,22}$. Como regra geral, tais estudos têm evidenciado elevadas prevalências da deficiência em nosso meio. No Município de São Paulo, em particular, estudo realizado na primeira metade da década de 70 indicou ser a anemia importante problema de saúde para a população de crianças menores de cinco anos, inclusive para aquelas pertencentes a famílias de melhor nível socioeconômico ${ }^{20}$.
O presente artigo descreve resultados de amplo estudo epidemiológico sobre condições de saúde e nutrição na infância realizado no Município de São Paulo, em $1984 / 85$, focalizando especificamente os achados do estudo relacionados à prevalência e distribuição da anemia.

\section{METODOLOGIA}

A amostra integral da pesquisa "Estudo das condições de saúde e nutrição das crinças do Município de São Paulo 1984/85" envolveu 1.016 crianças residentes nesse Município e com idades entre zero e 59 meses. O processo de amostragem adotado encontra-se descrito em artigo anterior desta série ${ }^{13}$, onde se demonstram o caráter probabilístico da amostra e a representatividade dos resultados gerados para o conjunto das crianças do Município estudado. Das 1.016 crianças da amostra $(89,8 \%), 912$ foram submetidas a exame hematológico para diagnóstico de anemia. A perda de cerca de $10 \%$ da amostra original foi devida parte à não concordância de familiares com o exame, parte a condições inadequadas de colheita de sangue. Analisadas com relação à faixa etária e nível socioeconômico (medido pelo ní-

* Realizado com auxílio financeiro da Financiadora de Estudos e Projetos - FINEP (Convênio: 4.1.83.0698.00) e Fundação de Amparo à Pesquisa do Estado de São Paulo - FAPESP (Processo 86/0392-7).

** Departamento de Nutrição da Faculdade de Saúde Pública da Universidade de São Paulo - Av. Dr. Arnaldo, 715 - 01255 São Paulo, SP - Brasil. 
vel de escolaridade do chefe da família), as crianças não submetidas a exame não se mostraram distintas das crianças examinadas (Prova de Kolmogorov-Smirnof, $\alpha$ $5 \%{ }^{19}$.

A colheita de sangue, feita no próprio domicilio da criança, foi executada através de puntura digital, observando-se as recomendações para a técnica proposta por Dallman e Siimes ${ }^{*}$. A concentração de hemoglobina nas amostras colhidas foi medida pelo método da cianometahemoglobina. O estado de anemia foi diagnosticado quando a concentração de hemoglobina acusou menos de $11 \mathrm{~g} / \mathrm{d} 1$, atendendo critério proposto pela Organização Mundial de Saúde ${ }^{16}$ para crianças de seis meses a seis anos (as crianças da amostra com menos de seis meses, 8,2\% da amostra, para as quais é extremamente complexo estabelecer-se um único nível crítico de hemoglobina, constituem grupo tratato à parte na discussão dos resultados). Quadros severos de anemia foram diagnosticados sempre que a concentração de hemoglobina foi inferior a $9,5 \mathrm{~g} / \mathrm{d} 1$.

A distribuição da prevalência da anemia foi estudada quanto à faixa etária da criança e quanto ao nível socioeconômico da família, este indicado pelo nível máximo de escolaridade obtido pelo chefe da unidade familiar. Em artigo anterior desta série ${ }^{13}$, fundamenta-se a escolha da escolaridade como marcador apropriado do nível socioeconômico das famílias estudadas. Testes baseados na distribuição do Qui quadrado foram utilizados para se avaliar o significado estatístico das associações observadas, adotando-se $\alpha$ de $5 \%{ }^{19}$.

\section{RESULTADOS}

A Figura apresenta a curva de distribuição dos valores médios de hemoglobina observados na amostra. Atentando-se para a curva esperada em populações não anêmicas, registrada na mesma Figura, verifica-se que a partir da idade de dois meses, os valores observados na amostra são claramente deficientes. Nota-se ainda que os maiores diferenciais com os valores esperados encontram-se entre as idades de 6 a 24 meses. A partir dos 24 meses, os diferenciais tendem a ser progressivamente menores.

As cifras relativas às prevalências da anemia e da anemia severa podem ser vistas na Tabela 1. Para o total da amostra, as referidas prevalências foram, respectivamente, de $35,6 \%$ e $14,7 \%$ (omitidas as crianças menores de seis meses, pouco se alteram aquelas prevalências: $35,7 \%$ e $14,6 \%$ ). Confirmando fato já indicado pelos valores médios da concentração de hemoglobina, as maiores prevalências de anemia são encontradas entre os 6 e os 24 meses de idade: neste período, mais da metade das crianças apresentam-se anêmicas, sendo metade dos casos de anemia severa. A partir dos 24 meses, a prevalência da anemia declina de forma linear com a idade, atingindo na faixa etária de 48 a 60 meses menos de um quinto das crianças examinadas. Igualmente declinante é a proporção de casos de anemia de maior severidade. A associação entre idade e anemia se mostra significativa $(\mathrm{p}<0,005)$ tanto quanto se considera a prevalência total de anemia como quando se considera a prevalência exclusiva de casos severos (a exclusão das crianças menores de seis meses não altera este resultado).

\section{TABELA 1}

Prevalência $(\%)$ de anemia em crianças de zero a 59 meses de idade do Município de São Paulo (1984/85).

\begin{tabular}{rccc}
\hline $\begin{array}{c}\text { Idade } \\
\text { (meses) }\end{array}$ & Amostra & $\begin{array}{c}\text { Anemia } \\
(\mathrm{Hb}<11 \mathrm{~g} / \mathrm{dl})\end{array}$ & $\begin{array}{c}\text { Anemia Severa } \\
(\mathrm{Hb}<9,5 \mathrm{~g} / \mathrm{dl})\end{array}$ \\
\hline $0 \vdash 6$ & 75 & 34,7 & 16,0 \\
$6 \vdash 12$ & 82 & 53,7 & 26,8 \\
$12 \vdash 24$ & 186 & 58,1 & 28,0 \\
$24 \vdash 36$ & 206 & 32,0 & 11,6 \\
$36 \vdash 48$ & 199 & 26,1 & 6,5 \\
$48 \vdash 60$ & 164 & 17,7 & 6,7 \\
\hline Total & 912 & 35,6 & 14,7 \\
\hline
\end{tabular}

A Tabela 2 apresenta as prevalências de anemia encontradas entre os cinco estratos socioeconômicos em que se dividiu a população (os dois estratos de maior nível socioeconômico, correspondentes aos níveis superior e colegial de escolaridade, foram agrupados em um único estrato em face da pequena expressão dos mesmos na amostra). Embora nenhum estrato apresente-se totalmente imune à anemia, pode-se notar que a prevalência da deficiência tende a ser menor nos estratos de melhor nível socioeconômico. Esta relação inversa é particularmente evidente para os casos severos de anemia, cuja prevalência no estrato de melhor nível socioeconômico é três vezes inferior à mesma prevalência encontrada no estrato extremo oposto $(7,8 \%$ contra $22,2 \%)$. A associação entre nível socioeconômico e anemia alcança significado estatístico apenas com relação aos casos severos de anemia $(p<0,05)$.

\section{DISCUSSÃO E CONCLUSÃO}

A partir de amostra representativa da população de menores de cinco anos residente no Município de São Paulo, verificou-se que mais de um terço das crianças desta localidade encontram-se anêmicas. É importante assinalar que o nível crítico de hemoglobina utilizado para caracterizar a presença do estado anêmico - $\mathrm{Hb}$ $<11 \mathrm{~g} / \mathrm{dl}$ - condiciona grande especificidade ao diagnóstico da anemia e anula a possibilidade de se estar superestimando a prevalência daquela deficiência na população ${ }^{22}$. De fato, critérios mais refinados para o diagnóstico da anemia, como a resposta positiva da concentração de hemoglobina à suplementação hematíni$\mathrm{ca}$, evidenciam que o critério $\mathrm{Hb}<11 \mathrm{~g} / \mathrm{dl}$ pode mesmo subestimar a prevalência da deficiência ${ }^{9,21}$. Se adotássemos, por exemplo, as proporções de respostas positivas encontradas na India por Singla e col. ${ }^{21}$ para

* Dallman e Siimes ${ }^{5}$ referem a possibilidade de substituir o sangue venoso por capilar no diagnóstico da anemia, sem prejuízo da qualidade do exame laboratorial, desde que observados determinados cuidados técnicos na colheita da amostra. Tais cuidados foram enfatizados no treinamento oferecido aos médicos responsáveis pela colheita de sanque e sempre que, por qualquer motivo, as recomendações não podiam ser observadas, a amostra colhida era excluída do estudo. 

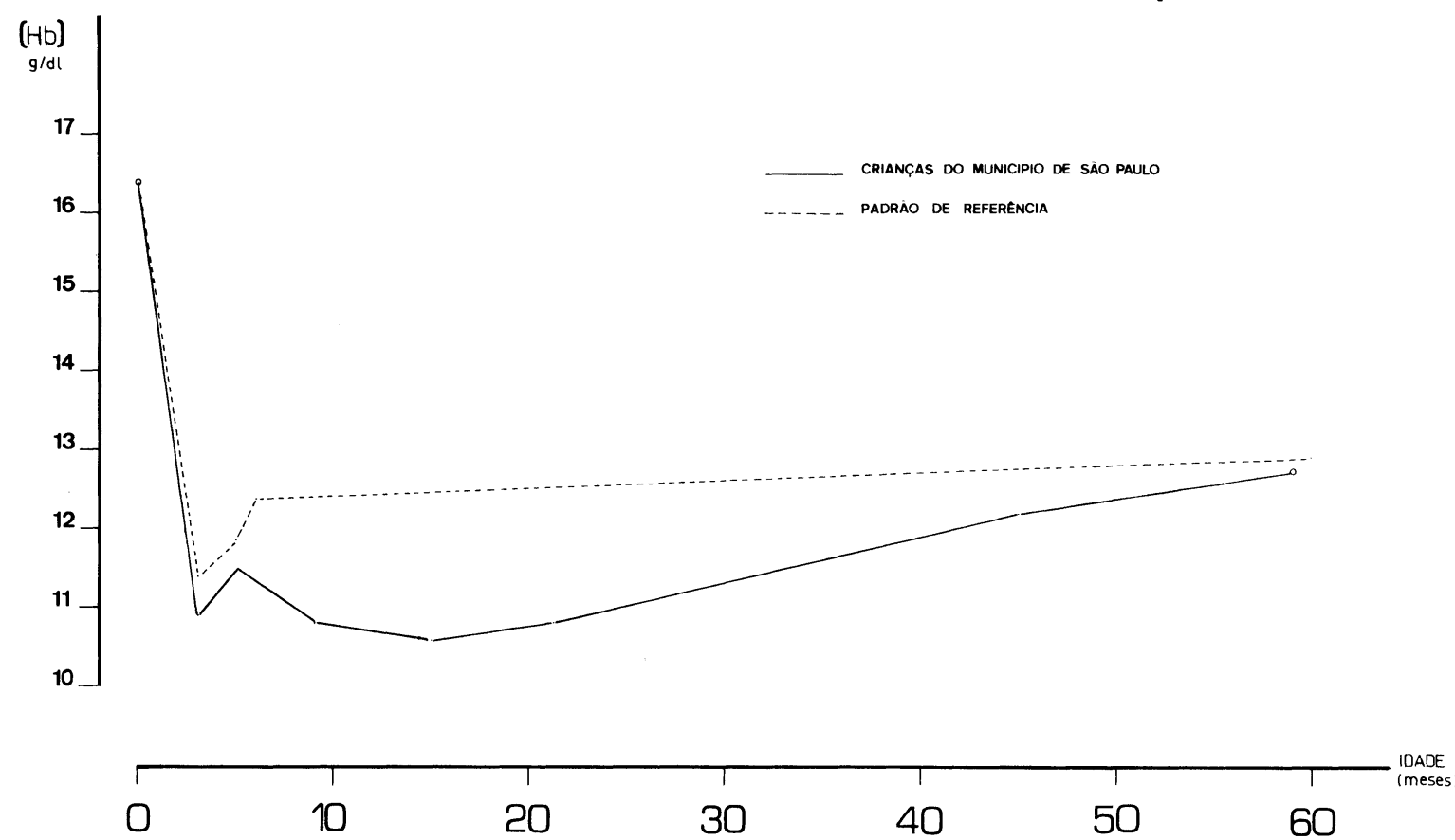

Figura - Padrão de referência proposto por Brault-Dubuc ${ }^{3}$ (zero a 36 meses) e por Dallman e Siimes ${ }^{4}$ ( 36 a 59 meses). Valores médios da concentração de hemoglobina nos primeiros cinco anos de vida. Município de São Paulo, $1984-1985$.

crianças com diferentes iniciais de hematócrito (inclusive com níveis correspondentes a concentrações de hemaglobina de $11 \mathrm{~g} / \mathrm{d} 1$ ou mais), chegaríamos à conclusão de que mais da metade das crianças estudadas em São Paulo responderiam à suplementação hematínica, o que demonstraria que a prevalência da deficiência em nosso meio seria ainda maior do que a estimada.

\section{TABELA 2}

Prevalência $(\%)$ da anemia entre crianças menores de cinco anos de diferentes estratos socioeconômicos do Município de São Paulo (1984/85).

\begin{tabular}{cccc}
\hline $\begin{array}{c}\text { Estrato } \\
\begin{array}{c}\text { Socio- } \\
\text { econômico* }\end{array}\end{array}$ & Amostra & $\begin{array}{c}\text { Anemia } \\
(\mathrm{Hb}<11 \mathrm{~g} / \mathrm{dl})\end{array}$ & $\begin{array}{c}\text { Anemia Severa } \\
(\mathrm{Hb}<9,5 \mathrm{~g} / \mathrm{dl})\end{array}$ \\
\hline I/II & 142 & 29,6 & 7,8 \\
III & 138 & 34,8 & 13,0 \\
IV & 296 & 36,5 & 15,2 \\
V & 240 & 35,4 & 16,7 \\
VI & 90 & 43,3 & 22,2 \\
\hline
\end{tabular}

* Estratos socioeconômicos constituídos a partir do nível de escolaridade do chefe da família da criança: $I=$ superior; $I I=$ colegial; $\mathrm{III}=$ ginasial; $\mathrm{IV}=$ primária completa; $\mathrm{V}=$ primária incompleta; VI = ausência de escolaridade.

Em relação à terminologia atualmente utilizada, os cursos primário, ginasial e colegial correspondem, respectivamente, às quatro primeiras séries do primeiro grau, às quatro últimas séries do primeiro grau e às três séries do segundo grau.

Observando-se os resultados encontrados pela mesma pesquisa "Estudo das condições de saúde e nutri- ção das crianças do Município de São Paulo" quanto à outra deficiência nutricional usualmente de grande expressão populacional - a desnutrição protéico-energética (DPE) - tem-se novo testemunho da importância da anemia na cidade de São Paulo. Medida pela prevalência de déficits ponderais - tal como proposto por Gomez e col. ${ }^{6}$ - a prevalência da DPE em São Paulo não chegaria a $30 \%{ }^{14}$; medida pela freqüência exclusiva de formas moderadas e severas, alternativa possivelmente mais apropriada para se estabelecer um paralelo correto com a freqüência da anemia, a prevalência da DPE não chegaria a $5 \%{ }^{14}$. Tais achados situam a anemia como deficiência nutricional inquestionavelmente mais comum entre as crianças do Município de São Paulo.

Outra forma de se apreciar a freqüência atual da anemia entre as crianças de São Paulo consiste em se comparar as prevalências observadas às prevalências registradas há cerca de dez anos (1973/74) por outro diagnóstico populacional realizado na cidade ${ }^{8}$. A referida comparação indica haver ocorrido no Município substancial elevação da prevalência da anemia: mais de $50 \%$ em relação à totalidade de casos e mais de $100 \%$ em relação às formas severas de anemia (Tabela 3). É importante assinalar que na comparação efetuada tomou-se o cuidado de omitir as crianças menores de seis meses, estudadas apenas em 1984/85, e de ajustar as prevalências do estudo de 1973/74 à real composição socioeconômica então existente na população do Município*. Em ambos os inquéritos a concentração de hemoglobina foi determinada pelo método de cianometahemoglobina. A diferente técnica de colheita utilizada, punção venosa no primeiro inquérito e puntura digital no segundo in-

* Tais ajustes, que pouco modificam os resultados, foram efetuados pelos autores deste trabalho levando em conta os fatores de correção utilizados por Batista Filho ${ }^{1}$ para estimar na mesma amostra de 1973/74 a real prevalência no Município de São Paulo, da DPE. 
quérito foram tomados todos os cuidados que a tornam comparáveis à punção venosa.

\section{TABELA 3}

Prevalência $(\%)$ de anemia entre crianças de 6 a 60 meses de idade em dois sucessivos inquéritos realizados no Município de São Paulo.

\begin{tabular}{cccc}
\hline Inquérito & Amostra & $\begin{array}{c}\text { Anemia } \\
(\mathrm{Hb}<1 \mathrm{~g} / \mathrm{dl})\end{array}$ & $\begin{array}{c}\text { Anemia Severa } \\
(\mathrm{Hb}<9,5 \mathrm{~g} / \mathrm{dl})\end{array}$ \\
\hline $1973 / 74$ & 278 & $22,7^{*}(23,1)^{* *}$ & $6,1^{*}(6,8)^{* *}$ \\
$1984 / 85$ & 837 & 35,5 & 14,6 \\
\hline
\end{tabular}

* Prevalências não ajustadas conforme Iunes e col. ${ }^{8}$

* * Prevalências ajustadas pelos autores deste trabalho levando em conta fatores de correção propostos por Batista Filho $^{1}$ (ver texto)

Acrescida das considerações acima, a atual magnitude alcançada pela anemia em São Paulo recomenda que as ações necessárias para seu controle sejam absolutamente priorizadas dentro dos programas de assistência à infância existentes na cidade. A este respeito, é interessante assinalar que ações exeqüíveis e relativamente eficazes no controle de anemia infantil - como o diagnóstico e tratamento sistemáticos, a suplementação medicamentosa preventiva em idades de maior risco, a nutrificação de determinados alimentos e a orientação adequada da alimentação do desmame - são incipientemente aplicadas em nosso meio, ou sequer são praticadas. Seria inteiramente desejável, neste particular, que a Secretaria de Saúde do Estado de São Paulo, que recentemente incorporou em sua programação ações de grande eficácia para o controle da DPE na infância (vigilância do crescimento, orientação nutricional e suplementação alimentar $)^{11}$, o mesmo fizesse com relação à anemia.

O estudo da distribuição da prevalência da anemia junto às diferentes faixas etárias da população evidencia que a idade da criança é elemento central na determinação do risco de ocorrência da deficiência. Com prevalências acima de 50\%, a faixa etária de 6 a 24 meses é a que condiciona os maiores riscos de anemia, devendo ser, portanto, o alvo preferencial de medidas de controle como a suplementação preventiva com sulfato ferroso. Igualmente evidente é a tendência de redução da anemia a partir da idade de dois anos, quando o aumento de idade passa a significar progressiva diminuição da vulnerabilidade infantil ao estado anêmico. É interessante mencionar que a intensa relação observada entre idade e anemia repete-se em outras pesquisas realizadas em nosso meio ${ }^{18,20,22}$.

A análise dos resultados referentes especificamente à faixa de zero a seis meses requer maiores cuidados, uma vez que a concentração normal de hemoglobina naquele período apresenta grandes variações. Comparandose, entretanto, a curva de variação dos valores médios de hemoglobina à curva que seria esperada no decorrer dos seis primeiros meses de vida (Figura), verifica-se que o risco de anemia está indiscutivelmente presente na população desde pelo menos a idade de dois meses. Em face de que nos primeiros meses de vida o organismo infantil encontra-se relativamente protegido da anemia, através dos mecanismos de re-utilização da hemoglobina fetal ${ }^{23}$, parece lícito inferir-se que poderosos condicionantes da anemia devam envolver as crianças de pequena idade no Município de São Paulo. Desde logo, é importante assinalar que as condições de prematuridade e/ou baixo peso ao nascer não explicam integralmente a precocidade do aparecimento da anemia em São Paulo, uma vez que é relativamente pequeną a incidência daquelas condições na cidade: $9,7 \%$ segundo levantamento realizado em maternidades ${ }^{10}$ e $8,7 \%$ de acordo com achados de nossa amostra de crianças.

Embora múltiplos fatores possam determinar o aparecimento da anemia na infância, usualmente dois são os fatores de maior expressão populacional: a presença de parasitas intestinais, sobretudo a ancilostomose, e o aporte dietético insuficiente de nutrientes, sobretudo de ferro $^{5,15}$.

Dados da mesma pesquisa de 1984/85* sugerem que as parasitoses intestinais não possam ser responsabilizadas pelas altas taxas de anemia observadas no Município de São Paulo: a ocorrência da ancilostomose na amostra de crianças estudadas foi desprezível (prevalência de $0,1 \%$ ); os demais parasitas alcançaram freqüências elevadas apenas entre as crianças mais velhas da amostra, as quais, como se viu, são as mais protegidas da anemia; a evolução da prevalência de todas as parasitoses intestinais foi favorável na última década, em franco contraste com o que se observa com relação a prevalência da anemia.

Análises preliminares das características da alimentação da mesma amostra de crianças estudadas em 1984/85* revelam que o fator dieta pode explicar em boa conta os achados relativos à ocorrência da anemia no Município de São Paulo: dietas deficientes em ferro foram encontradas em $43,6 \%$ das crianças estudadas, sendo ainda mais freqüente a deficiência nos primeiros dois anos de vida; o leite materno, excelente fonte de ferro de alta biodisponibilidade, só é majoritário na dieta infantil nos primeiros três meses de vida, sendo a partir desta idade substituído pelo leite de vaca integral, o qual, além de ser deficiente fonte de ferro, pode condicionar, quando em forma fluida, micro-hemorragias intestinais que atingirão sobretudo a criança de pequena idade ${ }^{15,17}$; o consumo de carne e feijão, importantes fontes de ferro na infância, além de ser expressivo apenas a partir

* Dados inéditos 
do segundo ano de vida, ocorre usualmente em quantidades demasiado restritas.

Os dados relativos à distribuição da prevalência da anemia pelos diferentes estratos socioeconômicos da população confirmam maior risco à doença a que, de modo geral, estão submetidas as famílias de pior nível socioeconômico da cidade ${ }^{12}$. É interessante, entretanto, notar que mesmo os estratos populacionais mais privilegiados da cidade se encontram submetidos a prevalências relativamente altas de anemia. Tal fato, que já havia sido observado em inquérito anterior realizado no Município $^{20}$, contrasta frontalmente com os achados da pesquisa 1984/85 referentes a outros eventos mórbidos, como a DPE ${ }^{14}$, a doença diarréica ${ }^{2}$ e as parasitoses intestinais ${ }^{12}$, que alcançam freqüências insignificantes ou nem mesmo ocorrem entre as famílias de melhor nível socioeconômico. Esta característica peculiar da anemia - sua disseminação por todos os estratos socioeconômicos da população - reforça a importância da deficiência em nosso meio, ao mesmo tempo que aponta para a necessidade de estudos mais refinados sobre sua epidemiologia. Neste sentido, parece fundamental que se conheça a exata determinação que a condição socioeconômica exerce sobre o aporte de ferro da dieta infantil, assim como os mecanismos que relacionam posteriormente este aporte às reservas de ferro e à concentração sangüínea da hemoglobina. Em trabalho futuro, voltaremos ao tema.

MONTEIRO, C.A. \& SZARFARC, S.C. [A study of children's health in S. Paulo city (Brazil), 1984-1985. V - Anemia]. Rev. Saúde públ., S. Paulo, 21:255-60, 1987.

ABSTRACT: As part of a broad epidemiologic survey on the health status of children living in the city of S.Paulo, Brazil, a representative sample of under-fives was studied with regard to the prevalence and distribution of anemia. The prevalence of anemic children (hemoglobin $<11 \mathrm{~g} / \mathrm{dl}$ ) was $35.6 \%$ and the prevalence of severe cases (hemoglobin $<9.5 \mathrm{~g} / \mathrm{dl}$ ) was $14.7 \%$. The highest rates of anemia were found between 6 and 24 months of age, a period in which more than half of the children surveyed were anemic and around $50 \%$ of the cases corresponded to severe forms of the deficiency. The prevalence of anemia tended to decrease with the improvement of the socioeconomonic status, but no segment of the population free from anemia was detected. Compared to the situation verified in the city around 10 years ago, an impressive increase in the prevalence of anemia was observed: of more than $50 \%$ for the total of anemic children and of more then $100 \%$ for the severe cases. In the light of all the data produced by the survey, there are strong evidences that child anemia in the city of S.Paulo is due rather to the unfavorable characteristics of the diet than to the presence of intestinal parasites.

UNITERMS: Child health. Health surveys. Anemia occurrence. Anemia, hypochromic, occurrence. Child nutrition. Socioeconomic factors.

\section{REFERÊNCIAS BIBLIOGRÁFICAS}

1. BATISTA FILHO, M. Prevalência e estágios da desnutrição protéico-calórica em crianças da cidade de São Paulo. São Paulo, 1976. [Tese de Doutorado - Faculdade de Saúde Pública da USP]

2. BENICIO, M.H. D'A. et al. Estudo das condições de saúde das crianças do Município de São Paulo, SP (Brasil), 1984-1985. IV - Doença diarréica. Rev. Saúde públ., S.Paulo, 21: 23-8, 1987.

3. BRAULT-DUBUC, M. et al. Iron status of French - Canadian children: a three year follow-up study. Hum. Nutr. appl.Nutr., 37A: 210-21, 1983.

4. DALlMAN, P.R. \& SIIMES, M.A. Percentiles curves for hemoglobin and red cell volume in infancy and childhood. J.Pediat., 94: 26-31, 1979.

5. DALLMAN, P.R. \& SIIMES, M.A. Iron deficiency in infant and childhood; a report of the International Nutritional Anemia Consultative Group (INACG). Washington, D.C., the Nutrition Foundation, 1979.

6. GOMES, F. et al. Mortality in second and third degree malnutrition. J.trop.Pediatr., 2: 77-83, 1956.
7. GANDRA, Y.R. Seminário sobre anemias nutricionais no Brasil; relatório final. Brasília, Instituto Nacional de Alimentação e Nutrição, 1977. [Mimeografado]

8. IUNES, E.S.M., coord. et al. Estado nutricional de crianças de 6 a 60 meses no Município de São Paulo: análise de dados. São Paulo, Instituto de Medicina Preventiva da Escola Paulista de Medicina/Instituto de Pesquisas Econômicas da USP, 1975. 2.v.

9. MARGOLIS, H.S. Iron deficiency in children: the relationship between pretreatment laboratory tests and subsequent hemoglobin response to iron therapy. Amer.J.clin. Nutr., 34: 2158-68, 1981.

10. MONTEIRO, C.A. et al. A distribuição do peso ao nascer no Município de São Paulo, Brasil. Rev.Saúde públ., S.Paulo, 14: 161-72, 1980

11. MONTEIRO, C.A., coord. et al. Recomendações sugeridas para o controle da desnutrição no Estado de São Paulo. São Paulo, Secretaria de Estado da Saúde, 1983. [Mimeografado]

12. MONTEIRO, C.A., coord. et al. Condições de saúde e nu- 
trição das crianças menores de cinco anos do Município de São Paulo; relatório final. São Paulo, Departamento de Nutrição da Faculdade de Saúde Pública da USP, 1986. [Mimeografado]

13. MONTEIRO, C.A. et al. Estudo das condições de saúde das crianças do Município de São Paulo, SP (Brazil), 1984-1985. I - Aspectos motodológicos, características sócio-econômicas e ambiente físico. Rev.Saúde públ., S.Paulo, 20: 435-45, 1986.

14. MONTEIRO, C.A. et al. Estudo das condições de saúde das crianças do Município de São Paulo, SP (Brazil), 1984-1985. II - Antropometria nutricional. Rev.Saúde públ. , S.Paulo, 20: 446-53, 1986.

15. NESTLÉ NUTRITION. El hierro en la alimentacion del lactante y del niño. [s.1.p.] 1984.

16. ORGANIZAÇÃO MUNDIAL DA SAÚDE. Grupo Científico de Anemias Nutricionales, Ginebra, 1968. Informe. Ginebra, 1968. (Ser.inf. técn., 405).

17. OSKI, F.A. Is bovine milk a health harzard? Pediatics, 75 (suppl.): 182-6, 1985.
18. SALZANO, A.C. et al. Anemias em crianças de dois serviços de saúde de Recife, PE (Brasil). Rev.Saúde públ., S.Paulo, 19: 499-507, 1985.

19. SIEGEL, S. Estatística não-paramétrica. São Paulo, Editora McGraw-Hill do Brasil, 1975.

20. SIGULEM, D.M. et al. Anemia ferropriva em crianças no Município de São Paulo. Rev.Saúde pùbl., S.Paulo, 12: 168-78, 1978.

21. SINGLA, P.N. et al. Deficiency anaemias in preschool children. Estimation of prevalence based on response to haematinic supplementation. J.trop.Pediat., 28: 77-82, 1982.

22. SZARFARC, S.C. Diagnóstico de dificiência de ferro na infância. Rev.Saúde públ., S.Paulo, 19: 278-84, 1985.

23. WOODRUFF, C.W. Iron deficiency in infancy and childhood; Symposium on Nutrition in Pediatrics. Pediat.clin.N.Amer., 24: 85-94, 1977.

Recebido para publicação em 04/2/1987 Aprovado para publicação em 27/4/1987 